\title{
Research of Influence of the Inlet Elements Design on a Hydraulic Efficiency of an Intermediate Stage in the BB3-Type Multi-Stage Centrifugal Pumps
}

\author{
A. S. Nadtochiy ${ }^{1 *}$ and I. B. Tverdohleb ${ }^{2}$ \\ 'JSC "HMS Livhydromash", Moscow branch, Russia \\ ${ }^{2}$ LLC "Management Company"HMS Group", Moscow, Russia
}

\begin{abstract}
This article presents the results of computational studies for different options of inlet design in an intermediate stage of a BB3 type multistage pump intended for transportation of crude oil and other similar liquids. Hydraulic losses in the flow part elements are compared for three different options of the inlet blades in the return channels of an intermediate stage.
\end{abstract}

\section{Introduction}

This work was carried out as part of a program on production of the BB3 type pumps. This refers to the volute centrifugal pumps with transfer channels that meet the requirements of GOST 32601-2013 [1].

These pumps are widely used as feed group pumps in the oil and gas refining, petrochemical and chemical industry, petroleum production, oil and oil products trunk transportation.

Since 2016, the enterprises of the HMS Group - JSC Sumy Nasosenergomash Plant and APOLLO - have been manufacturing and supplying the standard-size BB3 type pumps (figure 1).

\footnotetext{
* Corresponding author: nadtochiy@hms.ru
} 


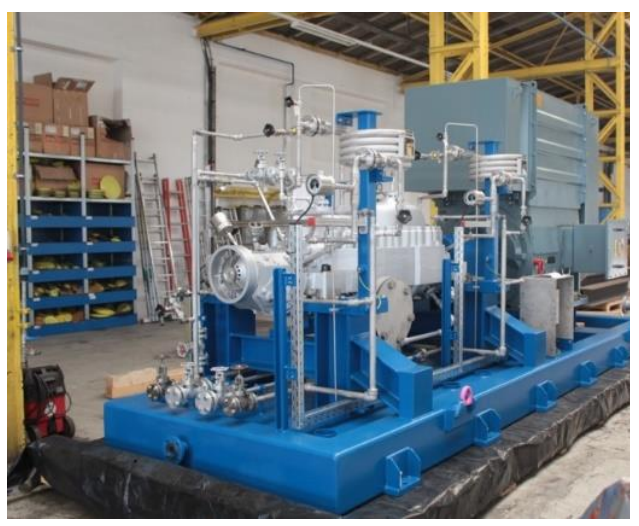

(a)

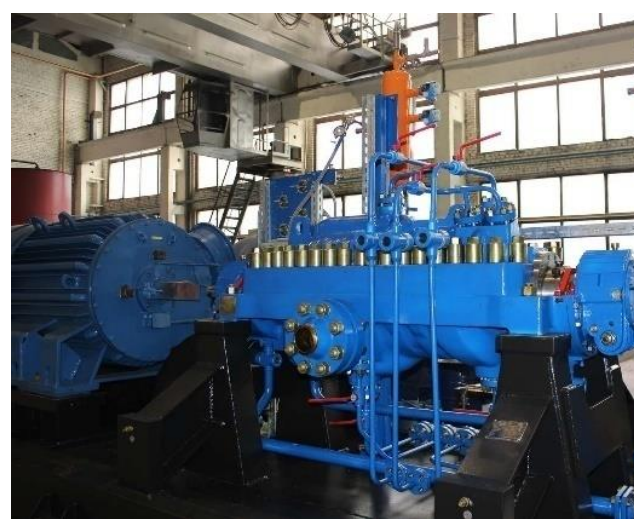

(b)

Fig. 1. AMG 150L.5x350 (a) and AMG 100L.13×290 (b) units.

The BB3 type pump with transfer channels between the groups of stages (figure 2) is a multistage, centrifugal, horizontal split pump, with "back-to-back" impeller configuration, which allows excluding the rotor full head balance device.

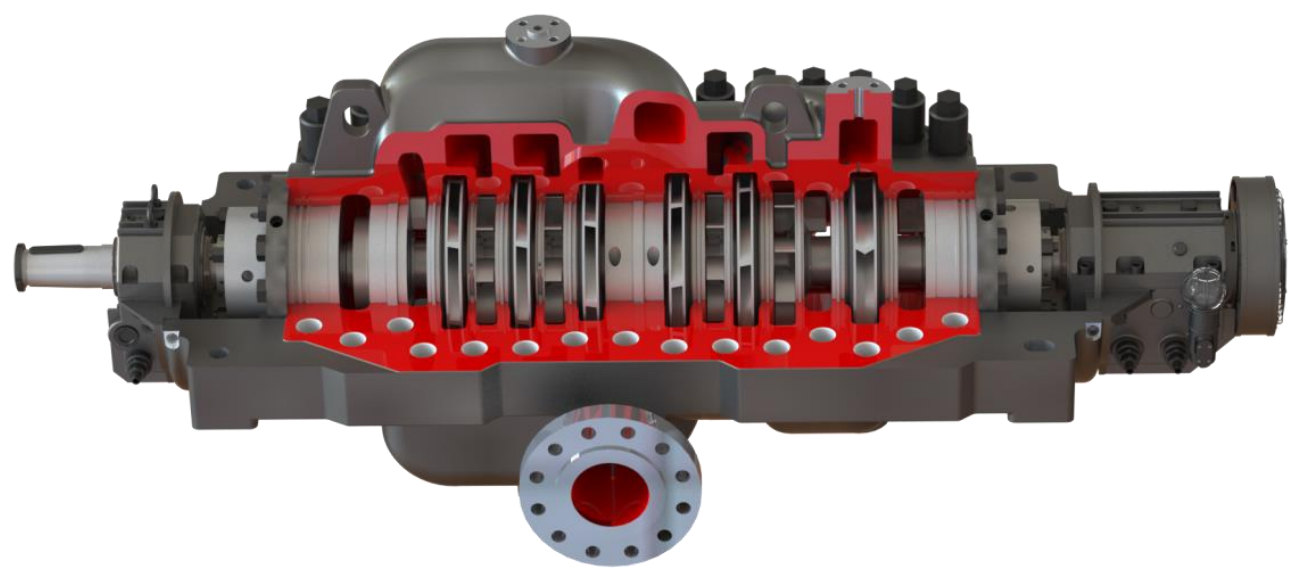

Fig. 2. BB3 type pump.

The return channels in the BB3 type pump are formed by an easily interchangeable element. A review of the literature [2,3] showed that there are no unambiguous recommendations regarding the design of the blades in the return channels. This article presents a part of the results of a study of fluid flow in the intermediate stage and shows the results for three types of blades in the return channels.

\section{Research methods}

Development of the operative parts of centrifugal pumps in order to increase the efficiency at a universal experimental bench tends to be a cost plus method which does not allow evaluating the mutual influence of structural components of the pump. Therefore, in this case, the most effective research method is a numerical experiment (NE). In this work, we used the PumpLinx software product by Simerics. 


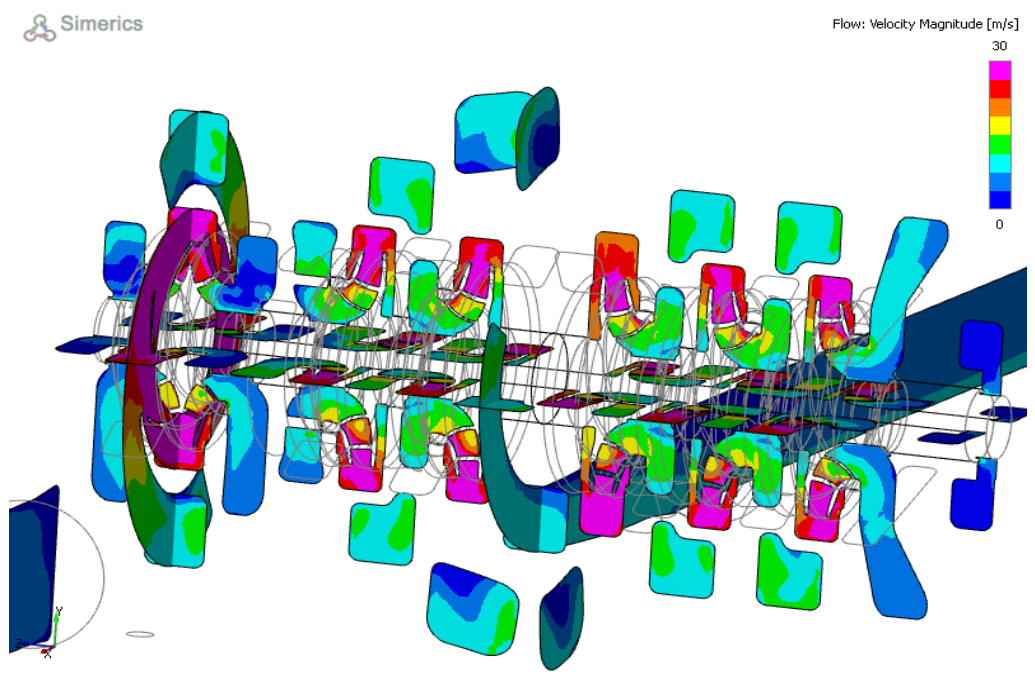

Fig. 3. Design model of a 6-stage BB3 type pump.

Figure 3 shows the model for a NE - the 6-stage pump. The components for research are shown in figure 4 . At the same time, the impeller sidewall gaps and neck rings are designed for the entire pump, and the surface roughness is set. Thus, this model allows one to obtain fairly accurate results without additional calculations of volumetric and mechanical losses. This article presents results for the $5^{\text {th }}$ intermediate stage only.

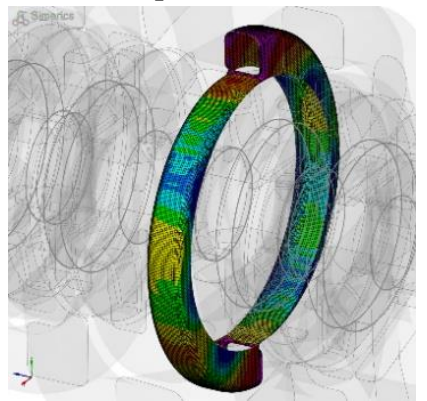

(a)

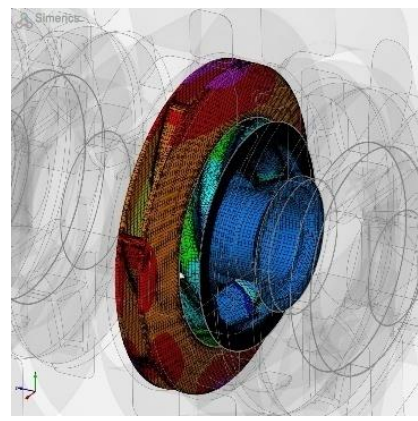

(d)

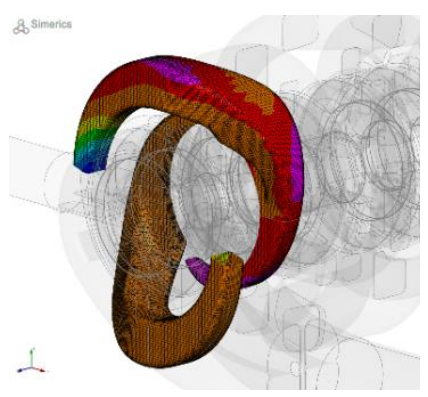

(b)

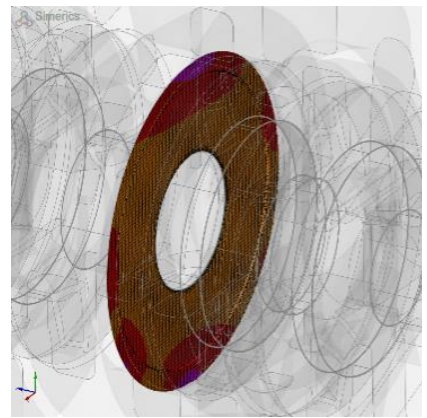

(e)

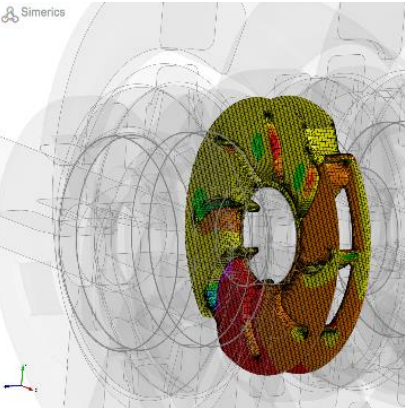

(c)

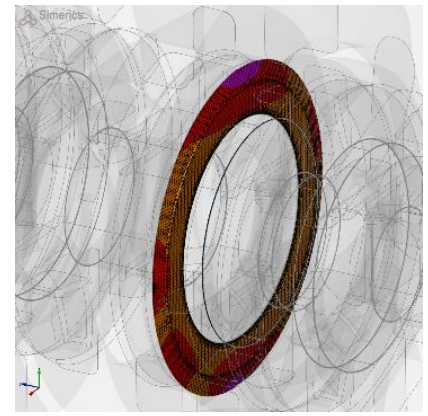

(f)

Fig. 4. Flow part components: outlet spiral components (a); transfer channels of the intermediate stage (b); intermediate stage outlet blades (c); impeller blades and inner plates (d); main plate (e); cover plate (f). 
The inlet pressure of 0 MPA was set. The flow rate was set at the outlet. The calculations were carried out under the unsteady mode with an angular step of rotation of $5^{\circ}$, because these results are more accurate, based on the experience of previous works and in comparison with the results obtained during the bench tests. The following boundary conditions were specified: $\mathrm{n}=2950 \mathrm{rpm}, \mathrm{t}=20^{\circ} \mathrm{C}, \rho=998 \mathrm{~kg} / \mathrm{m}^{3}$. The number of nodes in the grid for the entire model is $7.8 \mathrm{mln}$. The NE results are presented for 5 flow levels: 0.75 of the rated flow, 0.88 of the rated flow, rated flow, 1.13 of the rated flow, 1.25 of the rated flow.

\section{Research results and discussion}

One of the flow part (FP) components of the BB3 type pump, which affects the parameters and efficiency, is the inlet channel of the intermediate stage. The inlet channels in the BB3 type pump are formed by an easily interchangeable element. The following three types of interchangeable elements have been developed (figure 4):

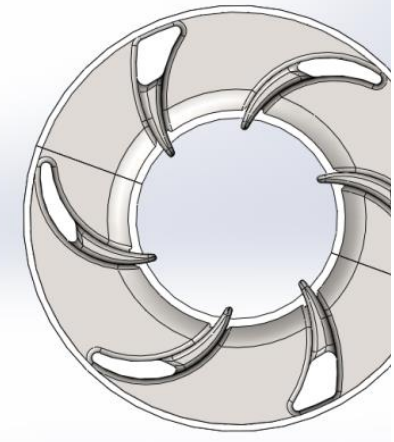

(a)

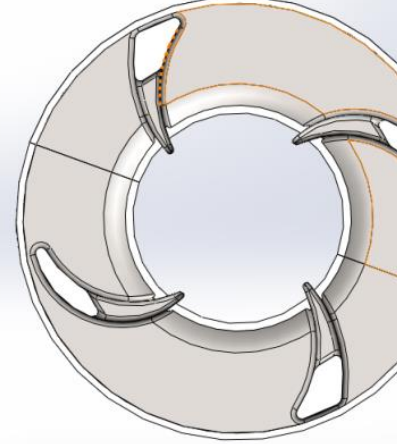

(b)

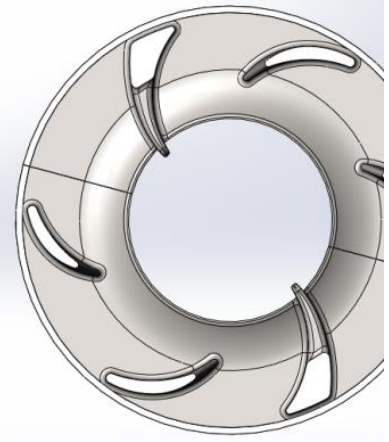

(c)

Fig. 5. Interchangeable elements of the inlet channels: $1^{\text {st }}$ option - component with 6 long blades (a); $2^{\text {nd }}$ option - component with 4 long blades (b); $3^{\text {rd }}$ option - component with 2 long and 4 short blades (c).

The analysis considered the flow in the inlet channels (figure 6) and upstream of the impeller (figure 7) and revealed the optimal impingement of the flow on the blades in the return channels for 3 inlet options.

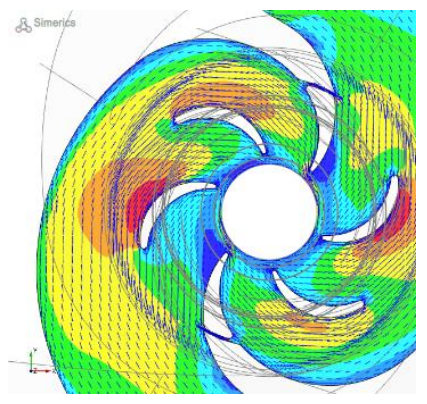

(a)

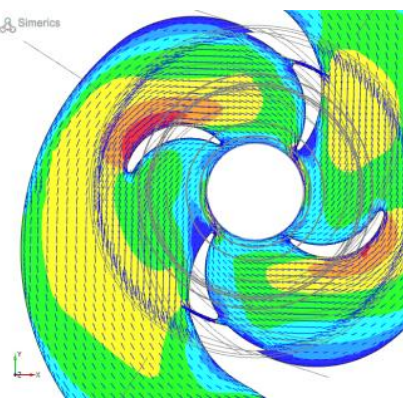

(b)

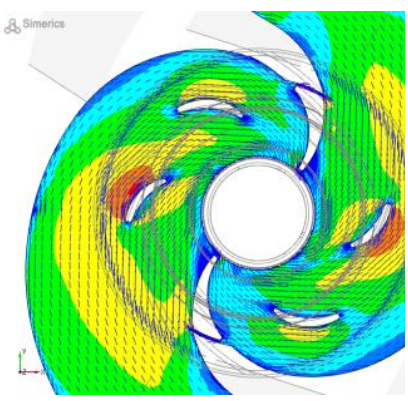

(c)

Fig. 6. Flow patterns and velocity distribution of the inlet channels at rated flow: $1^{\text {st }}$ option $-(a)$; $2^{\text {nd }}$ option $-(b) ; 3^{\text {rd }}$ option - (c). 


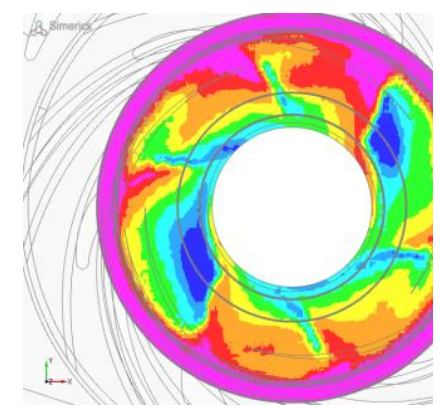

(a)

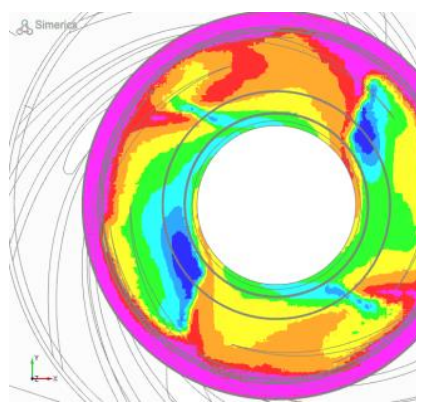

(b)

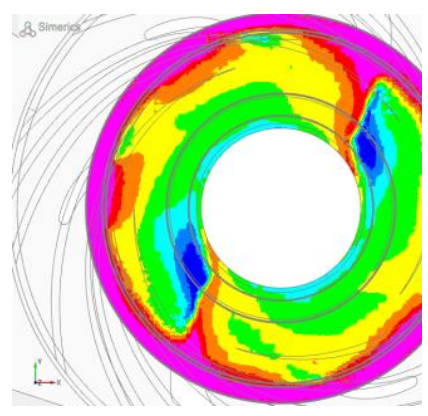

(c)

Fig. 7. Velocity distribution patterns upstream of the impeller at rated flow: $1^{\text {st }}$ option $-(a) ; 2^{\text {nd }}$ option - (b); $3^{\text {rd }}$ option - (c).

To estimate the flow upstream of the impeller, the flow angles of the fluid flow from the main plate to the cover plate were designed (figures 8-10) at the flow of 0.75 of the rated flow, rated flow, 1.25 of the rated flow. The velocity distribution pattern was also considered.

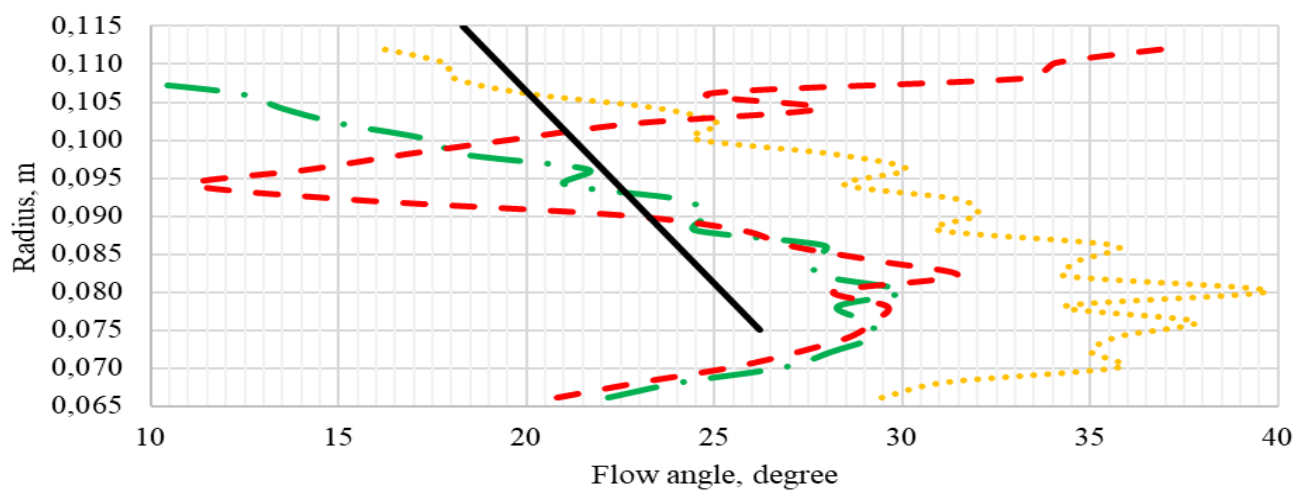

Fig. 8. The flow angles for the $1^{\text {st }}$ option $(0.75$ of the rated flow - red dashed curve, rated flow - green dash-and-dot curve, 1.25 of the rated flow - yellow dotted curve; impeller blade mounting angles black solid curve).

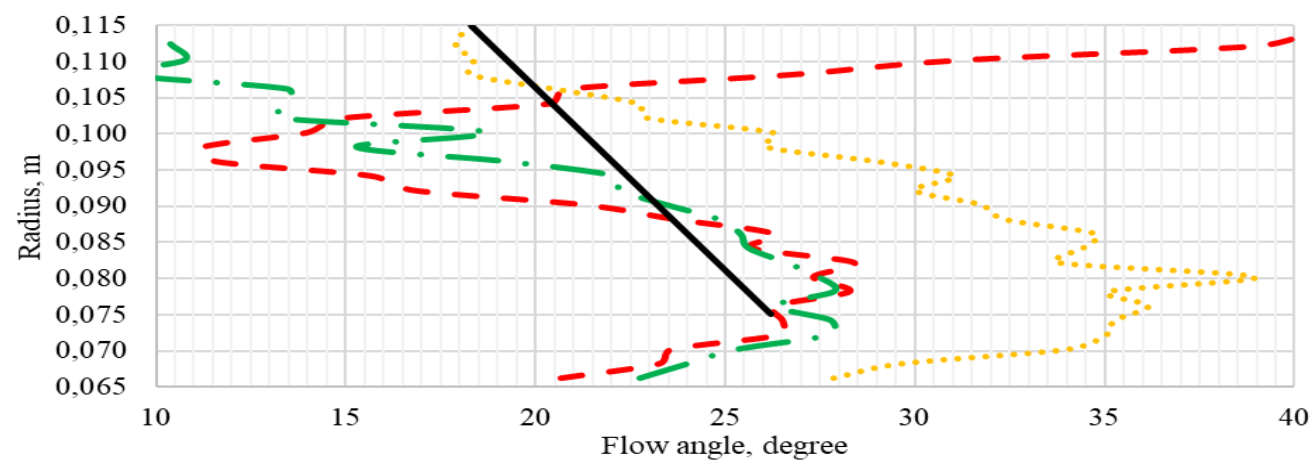

Fig. 9. The flow angles for the $2^{\text {nd }}$ option $(0.75$ of the rated flow - red dashed curve, rated flow - green dash-and-dot curve, 1.25 of the rated flow - yellow dotted curve; impeller blade mounting angles black solid curve). 


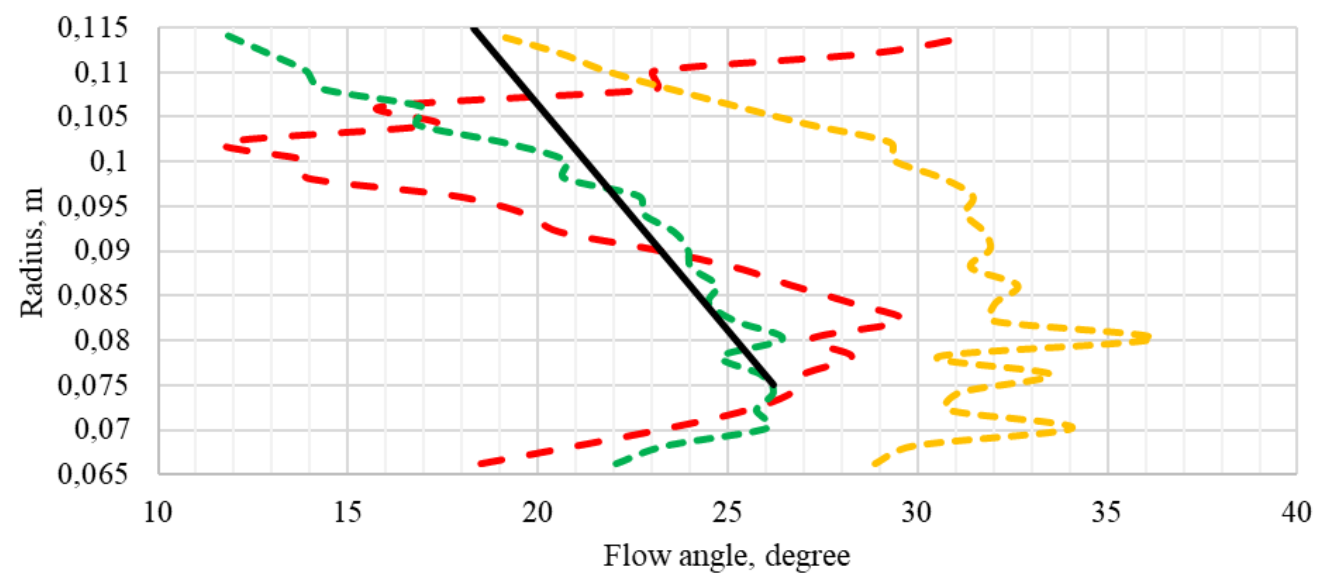

Fig. 10. The flow angles for the $3^{\text {rd }}$ option $(0.75$ of the rated flow - red dashed curve, rated flow - green dash-and-dot curve, 1.25 of the rated flow - yellow dotted curve; impeller blade mounting angles black solid curve).

Calculations showed that the best inlet element was $3^{\text {rd }}$ option, which has two long and four short blades. It proved minimal losses at the return blades (figure 11), as well as the maximum efficiency of the impeller - $94 \%$ (figure 12 ) due to the small angles of incidence at the blades (figure 10). Figure 13 shows that the maximum efficiency of the stage for $3^{\text {rd }}$ option is $82.9 \%$, for $1^{\text {st }}$ option $-81.8 \%$, and for $2^{\text {nd }}$ option $-82.3 \%$. Thus, the difference between the $2^{\text {nd }}$ and $3^{\text {rd }}$ option was $0.5 \%$. And between the $1^{\text {st }}$ and $3^{\text {rd }}$ option $-1.2 \%$, which is a good result for an interchangeable inlet element.

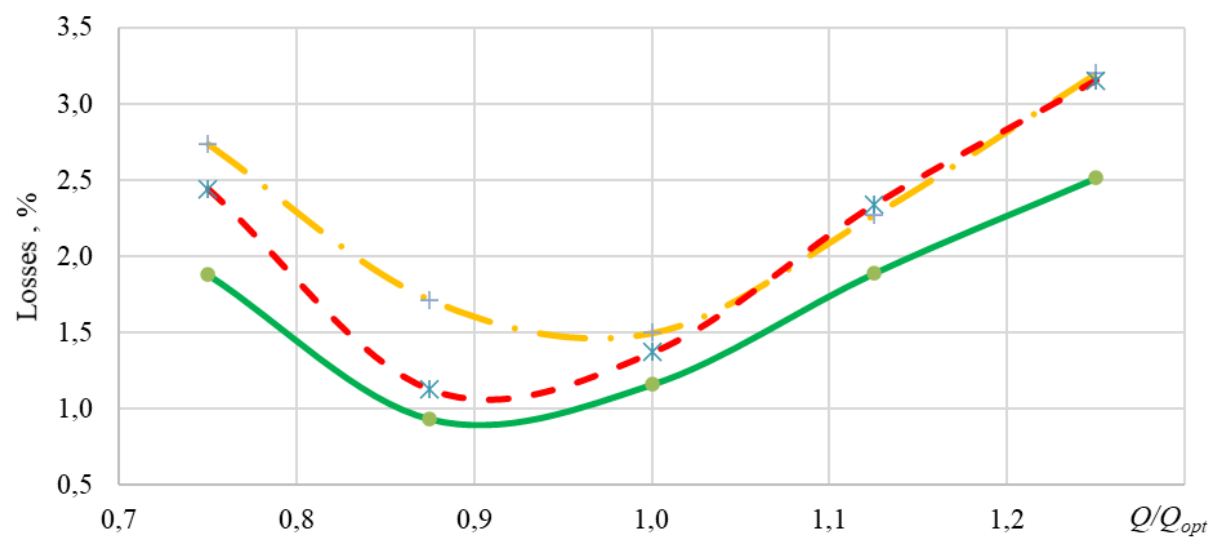

Fig. 11. Relative hydraulic losses at the inlet blades $\left(1^{\text {st }}\right.$ option - yellow dash-and-dot curve; $2^{\text {nd }}$ option - red dashed curve, $3^{\text {rd }}$ option - green solid curve). 


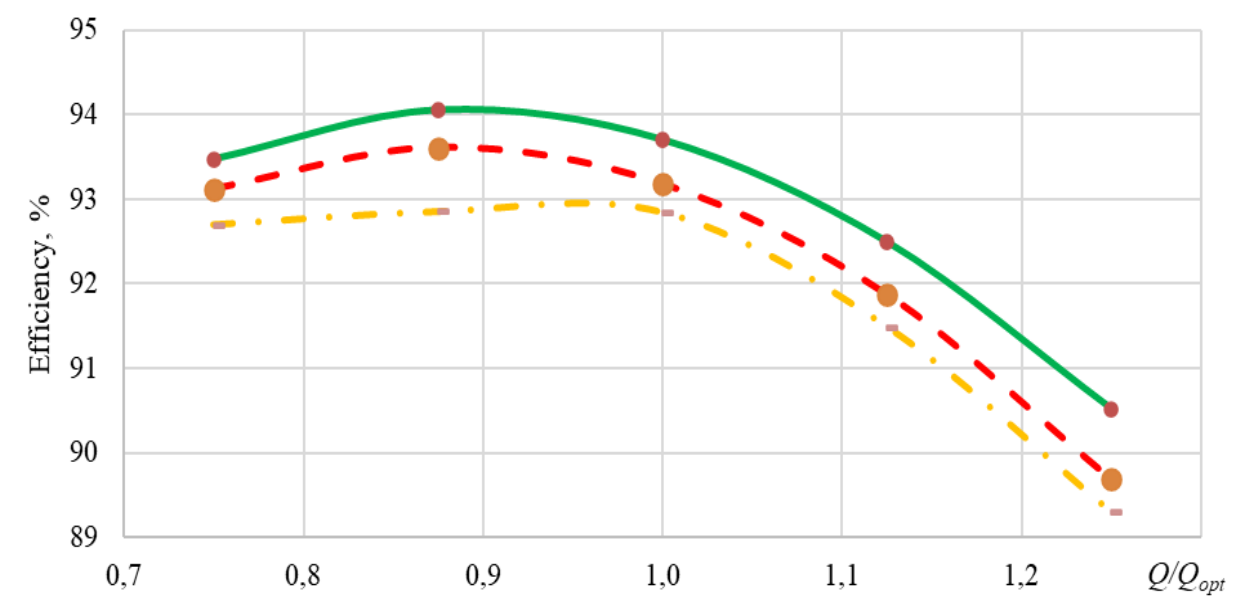

Fig. 12. Impeller efficiency ( $1^{\text {st }}$ option - yellow dash-and-dot curve; $2^{\text {nd }}$ option - red dashed curve, $3^{\text {rd }}$ option - green solid curve).

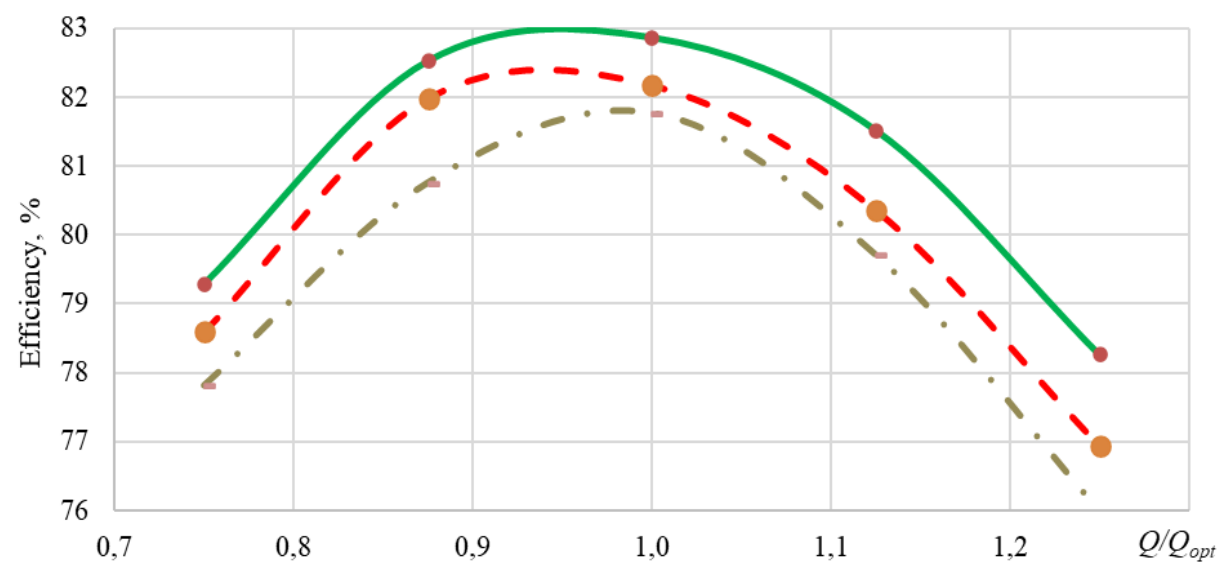

Fig. 13. Stage efficiency $\left(1^{\text {st }}\right.$ option - yellow dash-and-dot curve; $2^{\text {nd }}$ option - red dashed curve, $3^{\text {rd }}$ option - green solid curve).

\section{Conclusion}

The comparison of 3 types of inlet elements with different blade geometry was carried out. It is shown that with the purpose of losses minimization in the intermediate stage of the BB3 type pump the $3^{\text {rd }}$ interchangeable element is preferable, due to the following:

- Minimal hydraulic losses at the inlet element.

- Minimum angles of incidence at the impeller blades.

- The most uniform velocity distribution at the impeller inlet.

The results showed that the efficiency of the stage for the 3rd option is higher by 1.2 to $1.8 \%$ in comparison with other options for the inlet element design, and the maximum efficiency totaled to $82.9 \%$. Thus, the hydraulic efficiency of the developed stage with 2 long and 4 short blades in the return channels is comparable with world standards in pump engineering [2]. 


\section{References}

1. GOST 32601-2013, Centrifugal pumps for petroleum, petrochemical and natural gas industries. General technical requirements (mod. ISO13709:2009) (Moscow: Standardinform)

2. J. F. Gülich, Centrifugal pumps (Villeneuve, Switzerland: Springer International Publishing), 2020

3. V. S. Lobanoff and R. R. Ross, Centrifugal Pumps: Design and Application (Elsevier Inc.), 1992

4. S. S. Rudnev, Fundamentals of the theory of blade grids (Moscow) p 78, 1976 